\title{
Educación Virtual: una revisión sistemática
}

Virtual Education: a systematic review

\section{Chavez Ramirez, Marlene}

Licenciada en Contabilidad, Universidad Cesar Vallejo, Lima, Perú. ORCID:0000-0001-9632-6272

https://scholar.google.es/citations?hl=es\&user=KdicByQAAAAJ

\section{Lucila Rojas Delgado}

Maestra en Gestión de Servicios de Salud, Universidad Cesar Vallejo, Lima, Perú. ORCID:0000-0002-4575-3722

https://scholar.google.es/citations?view_op=list_works\&hl=es\&user=dcBAIu8AAAAJ

\section{Anticona Valderrama, Daniela Milagros}

Maestra en administración de la educación, Universidad Cesar Vallejo, Lima, Perú. ORCID: 0000- 0002- 1189- 4789

https://scholar.google.es/citations?user=JL0muMIAAAAJ\&hl=es\&oi=ao

\section{Edith Delia Chavez Ramirez}

Maestra en Gestión Pública, Universidad César Vallejo, Lima, Perú edithdeli@gmail.com, ORCID: https://orcid.org/0000-0002-3483-0825

https://scholar.google.es/citations?hl=es\&authuser=1\&user=qnSQmTgAAAAJ

\section{Revista Iberoamericana de la Educación \\ Vol - Especial 12021 \\ e-ISSN: 2737-632x}

Resumen: El presente artículo muestra una revisión sistemática referente a la educación virtual, mediante la búsqueda de distintas investigaciones se realiza el análisis de diferentes contextos donde se visualizan diferentes escenarios de la educación. Se realizó el análisis de producciones científicas del 2018 al 2021. Mediante la metodología de revisión sistemática. Los documentos examinados ayudan con datos empíricos y teóricos en la educación virtual. Se puede observar de los resultados que la educación virtual es adaptable en distintos contextos y se viene aplicando con éxito en la realidad de pandemia en la que vivimos. Palabras clave: Educación virtual, virtualidad, pandemia, contextos educativos. 
Abstract: This article shows a systematic review regarding virtual education, through the search for different investigations, the analysis of different contexts is carried out where different educational scenarios are visualized. The analysis of scientific productions from 2018 to 2021 was carried out. Using the systematic review methodology. The documents examined help with empirical and theoretical data in virtual education. It can be seen from the results that virtual education is adaptable in different contexts and has been successfully applied in the pandemic reality in which we live. Keywords: Virtual education, virtuality, pandemic, educational contexts.

\section{Introducción}

La educación es un indicador de desarrollo socioeconómico, de cara a una realidad pandémica a lo largo del último año se ha visto la necesidad de utilizar una técnica de estudio digitalizada como eje principal de aprendizaje, el uso de herramientas virtuales y tecnológicos ha sido fundamental para poder llegar a los alumnos y continuar una enseñanza; posteriormente se ve la necesidad de adaptar la educación virtual y aplicar estrategias para lograr que los alumnos tengan un aprendizaje (Chinchay, et. al, 2020). La educación virtual ha transformado la forma de enseñar y ha incrementado la investigación y el desarrollo de las herramientas pedagógicas, especialmente en como replantear el proceso de enseñanza aprendizaje y como desarrollar de manera rápida las competencias tecnológicas en los docentes y padres de familia para guiar a sus hijos. Se puede observar que los estudiantes se adecuan a la tecnología ágilmente, ya que muchos pertenecen a la era digital (Prata, Barbato y Gonzalez, 2020).

Este escenario ha conllevado a un reajuste en el ámbito educativo, la mayoría de instituciones no se encontraban preparadas para una experiencia similar, 
no estaba planificada específicamente, puesto que no había ocurrido algo similar. (Hodges et al., 2020). La pandemia ha expuesto la necesidad de transformación que requieren los sistemas educativos tradicionales y la importancia de tener estrategias educativas para el entorno virtual, destrezas y capacidades para la enseñanza y el aprendizaje en dicho entorno. (Tejedor, et al, 2020) Por ello cabe destacar la importancia de los ambientes virtuales que han sido de gran ayuda y han permitido continuar con la formación y originado evoluciones en los usos y funciones de las herramientas pedagógicas, el empleo de estas ha generado modos de comunicación, actuaciones e interpretaciones que modifican la percepción tiempo-espacio y la forma como los estudiantes originan su identidad docente. (Prata, Barbato y Gonzalez, 2020).

La educación virtual es la respuesta de la masificación y la expansión de la sociedad del conocimiento, este tipo de educación es un modelo competitivo a nivel regional, nacional e internacional que requiere la implementación de procesos académicos, tecnológicos y administrativos, y que permitan ofrecer formación de calidad desde cualquier lugar y tiempo. (Díaz et al, 2021) Así también las instituciones educativas deben considerar los lineamientos y los procesos tecno-pedagógicos desde una perspectiva diferente al tradicional discurso pedagógico, por lo contrario reconociendo el diseño objetos de aprendizaje y creación de ambientes de aprendizaje caracterizados por la interacción y comunicación que permitan gestionar, distribuir, evaluar y apoyar las diversas actividades de formación. (Basantes, 2018, p.36). Los Ambientes Virtuales de Aprendizaje (AVA) suscitan el desarrollo de estrategias metodológicas legítimas para promover el aprendizaje del alumnado bajo la orientación de un docente quien debe efectuar una conveniente planeación y diseño de su ambiente virtual de aprendizaje. (Cano, Domínguez y Ricardo, 2018) Al mismo tiempo, es importante 
establecer estrategias didácticas para lograr aprendizajes significativos caracterizados por la interactividad entre los participantes, estas se apoyan en varios medios, mediante el trabajo individual y el trabajo en equipo. (Rodríguez y Juanes, 2021).

Los recursos tecnológicos se han implicado cada vez más en los ambientes de formación, surgiendo nuevas inquietudes referentes con el uso conveniente de dichas herramientas, buscando lograr resultados positivos en el proceso enseñanza - aprendizaje, pretendiendo optimizar la calidad de la formación de los participantes. (Navarrete et al, 2018) En muchos países, la educación virtual requiere de estándares mínimos, está sujeta a evaluación y acreditación. En México, Venezuela y Colombia el enfoque por competencia es reducido, pero se observa el inicio de procesos de enseñanza virtuales en su totalidad y el surgimiento de aplicaciones informáticas enfocadas en sesiones prácticas y la adquisición de competencias. (Freire, Vera y Díaz, 2019). En el ámbito de la educación médica también se produjo la interrupción necesaria de las sesiones presenciales, lo que originó un debate y un dilema sobre las mejores acciones a seguir para impedir un desenlace negativo a largo plazo en la formación del futuro médico. (Vergara et al, 2020) La presente investigación pretende realizar una revisión sistemática a fin de evaluar la educación virtual en el Perú, para lo cual se ha considerado 21 investigaciones a nivel nacional e internacional. (Barros Bastidas \& Turpo Gebera, 2018)

\subsection{Variable de Estudio: Educación virtual}

En los estudios se visualiza las distintas problemáticas, teorías, metodologías relacionadas a la educación virtual, en la que se concluye que la educación virtual está respondiendo de manera exitosa en diferentes contextos. En la 
tabla No 1, se muestra el tema estudiado, autor, el año del estudio y las variables que se incluyen en la investigación. (Aguirre et al., 2019)

Tabla No. 1. Variable

\begin{tabular}{|c|c|c|c|c|}
\hline Tema & Autor & Año & País & Variables \\
\hline $\begin{array}{l}\text { Redes como espacio } \\
\text { de comunicación } \\
\text { para la educación } \\
\text { virtual de } \\
\text { estudiantes con } \\
\text { discapacidad en } \\
\text { Costa Rica en } \\
\text { tiempos de } \\
\text { pandemia }\end{array}$ & $\begin{array}{l}\text { Deliyore Vega, } \\
\text { María del Rocío }\end{array}$ & 2021 & Costa Rica & $\begin{array}{l}\text { - Discapacidad } \\
\text { - Pandemia } \\
\text { - Educación virtual } \\
\text { - Redes sociales } \\
\text { - Comunicación }\end{array}$ \\
\hline $\begin{array}{l}\text { Análisis del proceso } \\
\text { metodológico en } \\
\text { programas de } \\
\text { educación superior } \\
\text { en modalidad } \\
\text { virtual }\end{array}$ & $\begin{array}{l}\text { Díaz-Guillen, } \\
\text { Paula Andrea } \\
\text { Andrade-Arango, } \\
\text { Yamilhet } \\
\text { Hincapié-Zuleta, } \\
\text { Ana María } \\
\text { Uribe-Uran, } \\
\text { Adriana Patricia }\end{array}$ & 2021 & Colombia & $\begin{array}{l}\text { - Educación superior } \\
\text { - Modalidad virtual } \\
\text { - Proceso } \\
\text { metodológico } \\
\text { - Calidad } \\
\text { - Inclusión digital }\end{array}$ \\
\hline $\begin{array}{l}\text { Implementación de } \\
\text { una estrategia } \\
\text { didáctica para la } \\
\text { interactividad en } \\
\text { ambientes virtuales } \\
\text { para el posgrado en } \\
\text { la Educación } \\
\text { Superior }\end{array}$ & $\begin{array}{l}\text { Cinthya Rodríguez } \\
\text { Hernández } \\
\text { Blas Yoel Juanes } \\
\text { Giraud }\end{array}$ & 2021 & Cuba & $\begin{array}{l}\text { - Estrategia didáctica } \\
\text { - Interactividad } \\
\text { - En ambientes } \\
\text { virtuales } \\
\text { - Posgrado }\end{array}$ \\
\hline $\begin{array}{l}\text { Perspectivas de la } \\
\text { Educación Superior } \\
\text { en los Entornos } \\
\text { Virtuales en Perú }\end{array}$ & $\begin{array}{l}\text { Susana Soledad } \\
\text { Chinchay } \\
\text { Villarreyes, Luz } \\
\text { Arelis Moreno- } \\
\text { Quispe, Abraham } \\
\text { Guillermo } \\
\text { Ygnacio Santa } \\
\text { Cruz, José Jorge }\end{array}$ & 2021 & Perú & $\begin{array}{l}\text { - Entornos virtuales } \\
\text { - Educación Superior } \\
\text { - Universidades }\end{array}$ \\
\hline
\end{tabular}




\begin{tabular}{|c|c|c|c|c|}
\hline & $\begin{array}{l}\text { Zerga Romaní, } \\
\text { Juan Isaías Cango } \\
\text { Córdova }\end{array}$ & & & \\
\hline $\begin{array}{l}\text { Ambientes virtuales } \\
\text { de aprendizaje y } \\
\text { producción de } \\
\text { identidad en la } \\
\text { formación inicial } \\
\text { docente }\end{array}$ & $\begin{array}{l}\text { Danilo Prata, } \\
\text { Silviane Barbato, } \\
\text { María-Fernanda } \\
\text { González }\end{array}$ & 2020 & Brasil & $\begin{array}{l}\text { - Identidad docente } \\
\text { - Posicionamiento } \\
\text { - Enseñanza híbrida } \\
\text { - Educación superior }\end{array}$ \\
\hline $\begin{array}{l}\text { Adaptación de las } \\
\text { Metodologías } \\
\text { Activas en la } \\
\text { Educación } \\
\text { Universitaria en } \\
\text { Tiempos de } \\
\text { Pandemia }\end{array}$ & $\begin{array}{l}\text { Gómez-Hurtado, } \\
\text { I., García- } \\
\text { Rodríguez, M. del } \\
\text { P., González } \\
\text { Falcón, I., \& } \\
\text { Coronel Llamas, J. } \\
\text { M. }\end{array}$ & 2020 & España & $\begin{array}{l}\text { - Metodologías } \\
\text { activas } \\
\text { - Covid-19 } \\
\text { - Justicia social } \\
\text { - Metodología } \\
\text { cualitativa } \\
\text { - Educación } \\
\text { universitaria }\end{array}$ \\
\hline $\begin{array}{l}\text { Implementation } \\
\text { barriers in virtual } \\
\text { education in } \\
\text { Payame Noor } \\
\text { University in Iran }\end{array}$ & $\begin{array}{l}\text { Ahmadi, J } \\
\text { Nourabadi, S }\end{array}$ & 2020 & Irán & $\begin{array}{l}\text { - Barreras de } \\
\text { implementación } \\
\text { - Educación virtual } \\
\text { - Universidad Payame } \\
\text { Noor }\end{array}$ \\
\hline $\begin{array}{l}\text { Educación médica a } \\
\text { distancia en tiempos } \\
\text { de COVID-19 }\end{array}$ & $\begin{array}{l}\text { Vergara de la } \\
\text { Rosa, Esteban; } \\
\text { Vergara Tam, } \\
\text { Rodrigo; Alvarez } \\
\text { Vargas, Mayita; } \\
\text { Camacho } \\
\text { Saavedra, Luis y } \\
\text { Gálvez Olortegui, } \\
\text { José }\end{array}$ & 2020 & Cuba & $\begin{array}{l}\text { - Educación médica } \\
\text { - Educación a distancia } \\
\text { - Infecciones por } \\
\text { coronavirus } \\
\text { - COVID-19 } \\
\text { - América Latina }\end{array}$ \\
\hline $\begin{array}{l}\text { Marco legal de la } \\
\text { educación virtual a } \\
\text { distancia hacia su } \\
\text { implementación en } \\
\text { el contexto } \\
\text { latinoamericano }\end{array}$ & $\begin{array}{l}\text { Roger Freire } \\
\text { Avilés1 } \\
\text {, Néstor Vera } \\
\text { Lucio1 } \\
\text {, Judith Díaz } \\
\text { Nava2 }\end{array}$ & 2020 & Ecuador & $\begin{array}{l}\text { - Educación virtual a } \\
\text { distancia } \\
\text { - Modalidad } \\
\text { - Marco legal } \\
\text { - Innovación. }\end{array}$ \\
\hline $\begin{array}{l}\text { Virtual education: } \\
\text { factors that } \\
\text { influence its }\end{array}$ & $\begin{array}{l}\text { Héctor Varas- } \\
\text { Meza, Wendolin } \\
\text { Suárez-Amaya, }\end{array}$ & 2020 & Venezuela & - Gestión universitaria \\
\hline
\end{tabular}




\begin{tabular}{|c|c|c|c|c|}
\hline $\begin{array}{l}\text { expansion in Latin } \\
\text { America. }\end{array}$ & $\begin{array}{l}\text { Carolina López- } \\
\text { Valenzuela, } \\
\text { Michel Valdés- } \\
\text { Montecinos }\end{array}$ & & & $\begin{array}{l}\text { - Educación a } \\
\text { distancia } \\
\text { - Educación superior }\end{array}$ \\
\hline $\begin{array}{l}\text { Educación en } \\
\text { tiempos de } \\
\text { pandemia: } \\
\text { reflexiones de } \\
\text { alumnos y } \\
\text { profesores sobre la } \\
\text { enseñanza virtual } \\
\text { universitaria en } \\
\text { España, Italia y } \\
\text { Ecuador }\end{array}$ & $\begin{array}{l}\text { Santiago Tejedor, } \\
\text { Laura Cervi, } \\
\text { Fernanda Tusa, } \\
\text { Alberto Parola. }\end{array}$ & 2020 & $\begin{array}{l}\text { España, } \\
\text { Italia y } \\
\text { Ecuador }\end{array}$ & $\begin{array}{l}\text { - Tecnología } \\
\text { educativa } \\
\text { - Educación Superior } \\
\text { - Educación pública } \\
\text { - Proceso educativo } \\
\text { - Adaptación } \\
\text { estudiantil } \\
\text { - Educación a } \\
\text { distancia } \\
\text { - Aprendizaje virtual }\end{array}$ \\
\hline $\begin{array}{l}\text { Entorno virtual para } \\
\text { cocrear recursos } \\
\text { educativos digitales } \\
\text { en la educación } \\
\text { superior }\end{array}$ & $\begin{array}{l}\text { Bell Manrique- } \\
\text { Losada, María I. } \\
\text { Zapata Cárdenas, } \\
\text { Sandra I. Arango } \\
\text { Vásquez }\end{array}$ & 2020 & Colombia & $\begin{array}{l}\text { - Recursos de } \\
\text { aprendizaje digital } \\
\text { - Recursos abiertos } \\
\text { - Ambientes de } \\
\text { aprendizaje } \\
\text { - Cocreación } \\
\text { - Contenidos de } \\
\text { aprendizaje } \\
\text { - Metodologías de } \\
\text { enseñanza- } \\
\text { aprendizaje. }\end{array}$ \\
\hline $\begin{array}{l}\text { El uso del Ambiente } \\
\text { Virtual de } \\
\text { Aprendizaje entre el } \\
\text { profesorado de } \\
\text { educación superior: } \\
\text { un análisis de } \\
\text { género. }\end{array}$ & $\begin{array}{l}\text { Annachiara Del } \\
\text { Prete y Julio } \\
\text { Cabero Almenara }\end{array}$ & 2020 & Chile & $\begin{array}{l}\text { - Brecha digital de } \\
\text { género } \\
\text { - Ambientes Virtual } \\
\text { de Aprendizaje } \\
\text { - Educación Superior } \\
\text { - Competencias } \\
\text { digitales }\end{array}$ \\
\hline $\begin{array}{l}\text { Un reto en tiempos } \\
\text { de pandemia para la } \\
\text { educación médica } \\
\text { en Cuba }\end{array}$ & $\begin{array}{l}\text { Sergio González- } \\
\text { García, Isis } \\
\text { Casadelvalle } \\
\text { Pérez, Marco } \\
\text { Octavio Urda, } \\
\text { Thorvald Fortún } \\
\text { Sampayo, } \\
\text { Natascha Mezquía }\end{array}$ & 2020 & Cuba & $\begin{array}{l}\text { - Educación médica } \\
\text { - COVID-19 } \\
\text { - SARSCov2 } \\
\text { - Pregrado } \\
\text { - Posgrado } \\
\text { - Educación a } \\
\text { distancia. }\end{array}$ \\
\hline
\end{tabular}




\begin{tabular}{|c|c|c|c|c|}
\hline & $\begin{array}{l}\text { de Pedro, Raquel } \\
\text { G. Melón } \\
\text { Rodríguez }\end{array}$ & & & \\
\hline $\begin{array}{l}\text { Tecnologias digitais } \\
\text { de comunicação e } \\
\text { ensino superior: } \\
\text { contribuições da } \\
\text { teoria da atividade } \\
\text { em processos de } \\
\text { ensino- } \\
\text { aprendizagem }\end{array}$ & $\begin{array}{l}\text { Mariano Castro } \\
\text { Neto }\end{array}$ & 2020 & Brasil & $\begin{array}{l}\text { - Teoría de la } \\
\text { actividad } \\
\text { - Procesos de } \\
\text { enseñanza- } \\
\text { aprendizaje } \\
\text { - Tecnologías de } \\
\text { comunicación digital }\end{array}$ \\
\hline $\begin{array}{l}\text { E-learning in } 15 \\
\text { days. Challenges } \\
\text { and renovations in } \\
\text { Primary and } \\
\text { Secondary } \\
\text { Education of the } \\
\text { Republic of Croatia } \\
\text { during the COVID- } \\
19 \text { crisis. How have } \\
\text { we Introduced } \\
\text { distance Learning? }\end{array}$ & $\begin{array}{l}\text { Alicia Sianes Bautista, } \\
\text { Encarnación Sánchez } \\
\text { Lissen }\end{array}$ & 2020 & $\begin{array}{l}\text { República } \\
\text { de Croacia }\end{array}$ & $\begin{array}{l}\text { - Tecnologías de la } \\
\text { Información y la } \\
\text { Comunicación } \\
\text { - Aprendizaje a } \\
\text { distancia } \\
\text { - COVID19 } \\
\text { - Competencias } \\
\text { digitales }\end{array}$ \\
\hline $\begin{array}{l}\text { M- learning, un } \\
\text { camino hacia } \\
\text { aprendizaje ubicuo } \\
\text { en la educación } \\
\text { superior del } \\
\text { Ecuador }\end{array}$ & $\begin{array}{l}\text { Gladys Gioconda } \\
\text { Lagos Reinoso, } \\
\text { Katherine Mora } \\
\text { Barzola, Digna Mejía } \\
\text { Caguana, Rosario } \\
\text { Peláez Lopez, Juan } \\
\text { Carlos Peláez Lopez } \\
\end{array}$ & 2019 & Ecuador & $\begin{array}{l}\text { - Aprendizaje } \\
\text { - Dispositivos móviles } \\
\text { - Enseñanza } \\
\text { Tecnología } \\
\text { - Ubicuidad }\end{array}$ \\
\hline $\begin{array}{l}\text { El E-learning como } \\
\text { herramienta para la } \\
\text { educación continua: } \\
\text { una propuesta a la } \\
\text { formación } \\
\text { profesional en el } \\
\text { Ecuador }\end{array}$ & $\begin{array}{l}\text { Ginger Navarrete } \\
\text { Mendieta1, } \\
\text { Catalina Shirle } \\
\text { Morán Solís2, } \\
\text { María de los } \\
\text { Ángeles Guamán } \\
\text { Coronel3, } \\
\text { Myriam Irlanda } \\
\text { Arteaga Marín4, } \\
\text { José Patricio } \\
\text { Torres Fernández5 }\end{array}$ & 2019 & Ecuador & $\begin{array}{l}\text { - Formación } \\
\text { - Educación a } \\
\text { distancia } \\
\text { - Educación de los } \\
\text { trabajadores } \\
\text { - Tecnología de la } \\
\text { información }\end{array}$ \\
\hline $\begin{array}{l}\text { Plataformas } \\
\text { digitales en la }\end{array}$ & $\begin{array}{l}\text { Arturo Secundino } \\
\text { Hernández Gómez }\end{array}$ & 2019 & México & $\begin{array}{l}\text { - Educación } \\
\text { - Plataforma }\end{array}$ \\
\hline
\end{tabular}




\begin{tabular}{|c|c|c|c|c|}
\hline $\begin{array}{l}\text { educación a } \\
\text { distancia en } \\
\text { México, una } \\
\text { alternativa de } \\
\text { estudio en } \\
\text { comunicación }\end{array}$ & $\begin{array}{l}\text { Ennio Héctor } \\
\text { Carro Pérez } \\
\text { Isaías Martínez } \\
\text { Trejo }\end{array}$ & & & $\begin{array}{l}\text { - En línea } \\
\text { - Abierto } \\
\text { - Virtual }\end{array}$ \\
\hline $\begin{array}{l}\text { Hacia un nuevo } \\
\text { concepto: } \\
\text { Plataformas } \\
\text { Virtuales } \\
\text { Socioformativas } \\
\text { (PVS) }\end{array}$ & $\begin{array}{l}\text { Bibiana TOBON, } \\
\text { Sergio TOBÓN, } \\
\text { María Guadalupe } \\
\text { VEYTIA- } \\
\text { BUCHELI, } \\
\text { Alexandro } \\
\text { ESCUDERO }\end{array}$ & 2018 & $\begin{array}{l}\text { Latinoame } \\
\text { rica }\end{array}$ & $\begin{array}{l}\text { - Plataformas e- } \\
\text { learning } \\
\text { - Plataformas virtuales } \\
\text { socioformativas } \\
\text { - Socioformación } \\
\text { - Tecnologías de la } \\
\text { Información y la } \\
\text { Comunicación }\end{array}$ \\
\hline $\begin{array}{l}\text { Fortalecimiento de } \\
\text { la competencia TIC } \\
\text { de estudiantes de } \\
\text { educación superior } \\
\text { en Ambientes } \\
\text { Virtuales de } \\
\text { Aprendizaje }\end{array}$ & $\begin{array}{l}\text { John CANO } \\
\text { Barrios; Anderson } \\
\text { DOMINGUEZ; } \\
\text { Carmen } \\
\text { RICARDO } \\
\text { Barreto }\end{array}$ & 2018 & Colombia & $\begin{array}{l}\text { - Competencia } \\
\text { - TIC } \\
\text { - Estudiantes } \\
\text { - Educación } \\
\text { - Superior } \\
\text { - Ambientes Virtuales } \\
\text { de aprendizaje }\end{array}$ \\
\hline
\end{tabular}

En los diversos artículos se puede rescatar sobre la educación virtual, que es un mecanismo fundamental de interacción social remota de las instituciones, así como de teletrabajo, telemedicina, trasmisión de noticias, en el diseño de productos y soluciones para todo tipo de empresa. (Herrera-Tapia y Navia, 2020). Hoy obtener una ventaja competitiva en una institución es gran medida gracias a la tecnología, se debe tener en cuenta las innovaciones tecnológicas, lo cual va a permitir aumentar la eficiencia y obtención de beneficios (Villegas, Walle-Vazquez y Álvarez-Aros, 2020), además es importante fortalecer las competencias tecnológicas y el conocimiento de las plataformas socioeducativas, tanto de los estudiantes como los docentes, para hacer del proceso enseñanza aprendizaje un procedimiento ágil, eficiente y eficaz, el donde se logre el objetivo educativo. 


\section{Metodología}

La investigación se fundamenta en la revisión y búsqueda documental, con parámetros de clasificación de las bases de datos como Scopus, Scielo y EBSCO. Se realizó las búsquedas de información mediante cadenas de búsqueda, definidas por cada base de datos de revistas indexadas, siendo estas Scielo (producción científica and year_cluster:( "2018" or "2019" or "2020" or "2021") and subject_area:("Educación Virtual") and type:("researcharticle") de igual manera en Scopus se usó TITLE-ABS-KEY (educación AND virtual) AND ( LIMIT-TO ( PUBYEAR , 2021 ) OR LIMIT-TO ( PUBYEAR, 2020 ) OR LIMIT-TO (PUBYEAR, 2019 ) OR LIMIT-TO ( PUBYEAR , 2018) AND ( LIMIT-TO ( DOCTYPE , "ar" ) ) AND ( LIMIT-TO ( SUBJAREA, "SOCI" ) ). Por otro lado, en EBSCO se realizó la búsqueda por keyword (educación virtual) AND (FM P) limit-to 2018010120211231, con el fin de categorizar los artículos se clasificó los distintos tipos de investigación con sus respectivas unidades de observación e instrumentos (se listan las investigaciones en relación al orden de autores de la tabla No. 1):

\begin{tabular}{|l|l|l|l|}
\hline \multicolumn{1}{|c|}{ Tema } & \multicolumn{1}{|c|}{$\begin{array}{c}\text { Tipos de } \\
\text { Investigación }\end{array}$} & $\begin{array}{c}\text { Unidades de } \\
\text { Observación }\end{array}$ & $\begin{array}{c}\text { Métodos e } \\
\text { instrumento }\end{array}$ \\
\hline $\begin{array}{l}\text { Redes como espacio de } \\
\text { comunicación para la } \\
\text { educación virtual de } \\
\text { estudiantes con } \\
\text { discapacidad en Costa Rica } \\
\text { en tiempos de pandemia }\end{array}$ & $\begin{array}{l}\text { enfoque } \\
\text { cualitativo }\end{array}$ & $\begin{array}{l}\text { docentes de } \\
\text { educación } \\
\text { especial y } \\
\text { terapia } \\
\text { ocupacional }\end{array}$ & Entrevista \\
\hline $\begin{array}{l}\text { Análisis del proceso } \\
\text { metodológico en programas } \\
\text { de educación superior en } \\
\text { modalidad virtual }\end{array}$ & $\begin{array}{l}\text { enfoque } \\
\text { cualitativo }\end{array}$ & $\begin{array}{l}\text { rectores, } \\
\text { docentes y } \\
\text { coordinadores } \\
10 \text { instituciones } \\
\text { de educación }\end{array}$ & Entrevista \\
\hline
\end{tabular}




\begin{tabular}{|c|c|c|c|}
\hline & & $\begin{array}{l}\text { superior en } \\
\text { Colombia }\end{array}$ & \\
\hline $\begin{array}{l}\text { Implementación de una } \\
\text { estrategia didáctica para la } \\
\text { interactividad en ambientes } \\
\text { virtuales para el posgrado } \\
\text { en la Educación Superior }\end{array}$ & $\begin{array}{l}\text { Pre } \\
\text { experimental }\end{array}$ & $\begin{array}{l}\text { Estudiantes de } \\
\text { posgrado }\end{array}$ & $\begin{array}{l}\text { Métodos } \\
\text { teóricos y } \\
\text { empíricos }\end{array}$ \\
\hline $\begin{array}{l}\text { Perspectivas de la } \\
\text { Educación Superior en los } \\
\text { Entornos Virtuales en Perú }\end{array}$ & $\begin{array}{l}\text { descriptiva y } \\
\text { documental }\end{array}$ & revisión teórico & $\begin{array}{l}\text { revisión } \\
\text { teórico }\end{array}$ \\
\hline $\begin{array}{l}\text { Ambientes virtuales de } \\
\text { aprendizaje y producción de } \\
\text { identidad en la formación } \\
\text { inicial docente }\end{array}$ & $\begin{array}{l}\text { enfoque } \\
\text { cualitativo }\end{array}$ & $\begin{array}{l}\text { estudiante de } \\
\text { formación } \\
\text { inicial docente }\end{array}$ & $\begin{array}{l}\text { estudio de } \\
\text { caso }\end{array}$ \\
\hline $\begin{array}{l}\text { Adaptación de las } \\
\text { Metodologías Activas en la } \\
\text { Educación Universitaria en } \\
\text { Tiempos de Pandemia }\end{array}$ & $\begin{array}{l}\text { enfoque } \\
\text { cualitativo }\end{array}$ & $\begin{array}{l}\text { estudiantes de } \\
\text { grado y } \\
\text { posgrado }\end{array}$ & $\begin{array}{l}\text { estudio de } \\
\text { casos } \\
\text { múltiple }\end{array}$ \\
\hline $\begin{array}{l}\text { Implementation barriers in } \\
\text { virtual education in Payame } \\
\text { Noor University in Iran }\end{array}$ & $\begin{array}{l}\text { enfoque } \\
\text { cualitativo }\end{array}$ & $\begin{array}{l}\text { Estudiantes de } \\
\text { educación } \\
\text { superior }\end{array}$ & \\
\hline $\begin{array}{l}\text { Educación médica a } \\
\text { distancia en tiempos de } \\
\text { COVID-19 }\end{array}$ & $\begin{array}{l}\text { enfoque } \\
\text { cualitativo }\end{array}$ & revisión teórico & $\begin{array}{l}\text { revisión } \\
\text { teórico }\end{array}$ \\
\hline $\begin{array}{l}\text { Marco legal de la educación } \\
\text { virtual a distancia hacia su } \\
\text { implementación en el } \\
\text { contexto latinoamericano }\end{array}$ & $\begin{array}{l}\text { Enfoque } \\
\text { cuantitativo, } \\
\text { Diseño } \\
\text { descriptivo }\end{array}$ & $\begin{array}{l}\text { coordinadores } \\
\text { de los centros de } \\
\text { educación } \\
\text { virtual }\end{array}$ & Cuestionario \\
\hline $\begin{array}{l}\text { Virtual education: factors } \\
\text { that influence its expansion } \\
\text { in Latin America. }\end{array}$ & $\begin{array}{l}\text { enfoque } \\
\text { cualitativo }\end{array}$ & $\begin{array}{l}\text { revisión } \\
\text { documental }\end{array}$ & $\begin{array}{l}\text { revisión } \\
\text { documental }\end{array}$ \\
\hline $\begin{array}{l}\text { Educación en tiempos de } \\
\text { pandemia: reflexiones de } \\
\text { alumnos y profesores sobre } \\
\text { la enseñanza virtual } \\
\text { universitaria en España, } \\
\text { Italia y Ecuador }\end{array}$ & $\begin{array}{l}\text { Diseño } \\
\text { descriptivo, } \\
\text { exploratorio y } \\
\text { explicativo }\end{array}$ & $\begin{array}{l}\text { Estudiantes y } \\
\text { docentes de } \\
\text { educación } \\
\text { superior }\end{array}$ & Cuestionario \\
\hline $\begin{array}{l}\text { Entorno virtual para cocrear } \\
\text { recursos educativos } \\
\text { digitales en la educación } \\
\text { superior }\end{array}$ & $\begin{array}{l}\text { Enfoque } \\
\text { cualitativo, } \\
\text { hermenéutico. }\end{array}$ & $\begin{array}{l}\text { Revisión } \\
\text { documental }\end{array}$ & $\begin{array}{l}\text { Revisión } \\
\text { documental }\end{array}$ \\
\hline
\end{tabular}




\begin{tabular}{|c|c|c|c|}
\hline $\begin{array}{l}\text { El uso del Ambiente Virtual } \\
\text { de Aprendizaje entre el } \\
\text { profesorado de educación } \\
\text { superior: un análisis de } \\
\text { género. }\end{array}$ & $\begin{array}{l}\text { Enfoque } \\
\text { Cuantitativo, } \\
\text { Diseño } \\
\text { descriptivo }\end{array}$ & $\begin{array}{l}\text { Docentes de } \\
\text { educación } \\
\text { superior }\end{array}$ & Cuestionario \\
\hline $\begin{array}{l}\text { Un reto en tiempos de } \\
\text { pandemia para la educación } \\
\text { médica en Cuba }\end{array}$ & $\begin{array}{l}\text { Enfoque } \\
\text { cualitativo }\end{array}$ & Revisión teórica & $\begin{array}{l}\text { Revisión } \\
\text { teórica }\end{array}$ \\
\hline $\begin{array}{l}\text { Tecnologias digitais de } \\
\text { comunicação e ensino } \\
\text { superior: contribuições da } \\
\text { teoria da atividade em } \\
\text { processos de ensino- } \\
\text { aprendizagem }\end{array}$ & $\begin{array}{l}\text { Enfoque } \\
\text { cualitativo, de } \\
\text { tipo exploratorio } \\
\text { comparativo }\end{array}$ & $\begin{array}{l}\text { Estudiantes y } \\
\text { docentes de } \\
\text { educación } \\
\text { superior }\end{array}$ & Cuestionario \\
\hline $\begin{array}{l}\text { E-learning in } 15 \text { days. } \\
\text { Challenges and renovations } \\
\text { in Primary and Secondary } \\
\text { Education of the Republic } \\
\text { of Croatia during the } \\
\text { COVID-19 crisis. How have } \\
\text { we Introduced distance } \\
\text { Learning? }\end{array}$ & $\begin{array}{l}\text { Enfoque } \\
\text { cualitativo }\end{array}$ & Revisión teórica & $\begin{array}{l}\text { Revisión } \\
\text { teórica }\end{array}$ \\
\hline $\begin{array}{l}\text { M- learning, un camino } \\
\text { hacia aprendizaje ubicuo en } \\
\text { la educación superior del } \\
\text { Ecuador }\end{array}$ & $\begin{array}{l}\text { Enfoque } \\
\text { Cuantitativo, } \\
\text { Diseño } \\
\text { descriptivo }\end{array}$ & $\begin{array}{l}\text { Estudiantes y } \\
\text { docentes de } \\
\text { educación } \\
\text { superior }\end{array}$ & Cuestionario \\
\hline $\begin{array}{l}\text { El E-learning como } \\
\text { herramienta para la } \\
\text { educación continua: una } \\
\text { propuesta a la formación } \\
\text { profesional en el Ecuador }\end{array}$ & $\begin{array}{l}\text { Enfoque } \\
\text { cualitativo, } \\
\text { diseño } \\
\text { exploratorio }\end{array}$ & $\begin{array}{l}\text { Empresas de } \\
\text { capacitación }\end{array}$ & $\begin{array}{l}\text { Entrevistas y } \\
\text { Observación }\end{array}$ \\
\hline $\begin{array}{l}\text { Plataformas digitales en la } \\
\text { educación a distancia en } \\
\text { México, una alternativa de } \\
\text { estudio en comunicación }\end{array}$ & $\begin{array}{l}\text { Enfoque } \\
\text { cualitativo, } \\
\text { transversal } \\
\text { descriptivo }\end{array}$ & $\begin{array}{l}\text { Revisión } \\
\text { documental }\end{array}$ & $\begin{array}{l}\text { Revisión } \\
\text { documental }\end{array}$ \\
\hline $\begin{array}{l}\text { Hacia un nuevo concepto: } \\
\text { Plataformas Virtuales } \\
\text { Socioformativas (PVS) }\end{array}$ & $\begin{array}{l}\text { Enfoque } \\
\text { cualitativo, } \\
\text { transversal } \\
\text { descriptivo }\end{array}$ & $\begin{array}{l}\text { Revisión } \\
\text { documental }\end{array}$ & $\begin{array}{l}\text { Revisión } \\
\text { documental }\end{array}$ \\
\hline
\end{tabular}




\begin{tabular}{|l|l|l|l|}
\hline $\begin{array}{l}\text { Fortalecimiento de la } \\
\text { competencia TIC de } \\
\text { estudiantes de educación } \\
\text { superior en Ambientes } \\
\text { Virtuales de Aprendizaje }\end{array}$ & $\begin{array}{l}\text { Enfoque } \\
\text { cuantitativo }\end{array}$ & $\begin{array}{l}\text { Estudiantes de } \\
\text { educación } \\
\text { superior }\end{array}$ & Cuestionario \\
\hline
\end{tabular}

En la tabla No 2 se observan los diversos tipos de metodología que son una gran contribución para el presente estudio, la categorización de diversos métodos e instrumentos necesarios en el procedimiento de recolección de información según el estudio de educación virtual. Además, se puede observar como actualmente la tecnología está inmersa en el ámbito educativo a nivel internacional y cómo han tenido que adaptarse muchos de estos países a las nuevas tendencias, muchas veces con recursos limitados. Los datos recogidos demostraron una comparación entre cada investigación, mostrando una mayor perspectiva del tema estudiado.

\section{Resultados}

En las investigaciones recolectadas los autores exponen sus resultados, contribuyendo a la comunidad científica con su aporte, es así se puede mostrar en la tabla No 3.

Tabla 3. Resultados

\begin{tabular}{|l|l|}
\hline \multicolumn{1}{|c|}{ Tema } & \multicolumn{1}{c|}{ Resultados } \\
\hline $\begin{array}{l}\text { Redes como espacio de } \\
\text { comunicación para la } \\
\text { educación virtual de } \\
\text { estudiantes con } \\
\text { discapacidad en Costa Rica } \\
\text { en tiempos de pandemia }\end{array}$ & $\begin{array}{l}\text { Busca proponer innovaciones tecnológicas para } \\
\text { favorecer a las personas con discapacidad, al ser una } \\
\text { experiencia compleja y en constante cambio, se } \\
\text { concluye que la coyuntura mundial frente a la pandemia } \\
\text { fomenta la innovación y creatividad docente lo que hace } \\
\text { que el estudiantado, logre familiarizarse y habituarse al } \\
\text { uso de redes de comunicación social, lo que facilita } \\
\text { tanto su aprendizaje como sus oportunidades de } \\
\text { inclusión social. }\end{array}$ \\
\hline
\end{tabular}




\begin{tabular}{|c|c|}
\hline $\begin{array}{l}\text { Análisis del proceso } \\
\text { metodológico en programas } \\
\text { de educación superior en } \\
\text { modalidad virtual }\end{array}$ & $\begin{array}{l}\text { Educación virtual resultado de los procesos de } \\
\text { globalización y de las innovaciones tecnológicas } \\
\text { Se permite el lenguaje digital para representar, simular y } \\
\text { recrear didácticamente la realidad sin limitaciones } \\
\text { espacio-temporales. Se busca la mejora de la calidad en } \\
\text { el proceso de educación virtual }\end{array}$ \\
\hline $\begin{array}{l}\text { Implementación de una } \\
\text { estrategia didáctica para la } \\
\text { interactividad en ambientes } \\
\text { virtuales para el posgrado en } \\
\text { la Educación Superior }\end{array}$ & $\begin{array}{l}\text { Se considera que los resultados en la implementación de } \\
\text { la estrategia didáctica demuestran lo significativo que } \\
\text { pueden ser los aprendizajes caracterizados por la } \\
\text { interactividad entre los participantes. }\end{array}$ \\
\hline $\begin{array}{l}\text { Perspectivas de la } \\
\text { Educación Superior en los } \\
\text { Entornos Virtuales en Perú }\end{array}$ & $\begin{array}{l}\text { Se concluye que la educación universitaria desde los } \\
\text { entornos virtuales transforma tecnológicamente la } \\
\text { relación educativa de un modo definido, de tal manera } \\
\text { que concede una actuación externa para el aprendizaje y } \\
\text { a partir de esa misma estructura y atributos } \\
\text { tecnológicos, promueve en el estudiante una } \\
\text { modificación interna de sus estrategias de pensamiento } \\
\text { y aprendizaje. }\end{array}$ \\
\hline $\begin{array}{l}\text { Ambientes virtuales de } \\
\text { aprendizaje y producción de } \\
\text { identidad en la formación } \\
\text { inicial docente }\end{array}$ & $\begin{array}{l}\text { Los resultados indican que las posiciones yo-otro- } \\
\text { mundo varían de acuerdo al ambiente virtual y a la } \\
\text { especificidad de las consignas de las actividades, y se } \\
\text { ponen en evidencia en las relaciones dialógicas en } \\
\text { múltiples planos } \\
\text { Se concluye que las actividades promueven nuevos } \\
\text { posicionamientos y que la identidad docente emerge en } \\
\text { el interjuego entre ser estudiante en el presente e ir } \\
\text { convirtiéndose en profesor. }\end{array}$ \\
\hline $\begin{array}{l}\text { Adaptación de las } \\
\text { Metodologías Activas en la } \\
\text { Educación Universitaria en } \\
\text { Tiempos de Pandemia }\end{array}$ & $\begin{array}{l}\text { Las metodologías activas utilizadas presencialmente se } \\
\text { han sustituido por otras estrategias y recursos didácticos } \\
\text { que han permitido una docencia virtual basada en un } \\
\text { proceso de enseñanza-aprendizaje activo y enriquecedor } \\
\text { para todos los estudiantes. }\end{array}$ \\
\hline $\begin{array}{l}\text { Implementation barriers in } \\
\text { virtual education in Payame } \\
\text { Noor University in Iran }\end{array}$ & $\begin{array}{l}\text { Los resultados mostraron que: Las principales barreras } \\
\text { para implementar la educación } \\
\text { sobre la utilización virtual son las barreras culturales. } \\
\text { Los hallazgos de este estudio indican que: La falta de } \\
\text { apoyo por parte } \\
\text { de los administradores universitarios, la falta de } \\
\text { información y capacitación útil para los estudiantes, y la } \\
\text { falta de }\end{array}$ \\
\hline
\end{tabular}




\begin{tabular}{|c|c|}
\hline & $\begin{array}{l}\text { conocimiento de las autoridades de educación superior } \\
\text { sobre el sistema educativo virtual son la primera } \\
\text { prioridad de las barreras de implementación para brindar } \\
\text { servicios virtuales. Educación. }\end{array}$ \\
\hline $\begin{array}{l}\text { Educación médica a } \\
\text { distancia en tiempos de } \\
\text { COVID-19 }\end{array}$ & $\begin{array}{l}\text { el análisis de las estrategias durante las epidemias } \\
\text { previas de SARS y MERS resalta la importancia de } \\
\text { tomar en cuenta acciones previas y adaptarlas a nuestra } \\
\text { realidad } \\
\text { la implementación de formas de aprendizaje virtual } \\
\text { (ABP) con mayor interacción con el docente, la } \\
\text { adaptación docente al uso de las TIC, el acceso } \\
\text { adecuado a una banda ancha, y una comunicación } \\
\text { estrecha con los alumnos y los padres de familia, para } \\
\text { tener el mayor número de herramientas virtuales que } \\
\text { permitan cubrir los diferentes estilos de aprendizaje. }\end{array}$ \\
\hline $\begin{array}{l}\text { Marco legal de la educación } \\
\text { virtual a distancia hacia su } \\
\text { implementación en el } \\
\text { contexto latinoamericano }\end{array}$ & $\begin{array}{l}\text { El marco legal está constituido por la Constitución de la } \\
\text { República Bolivariana de Venezuela } \\
\text { La educación virtual a distancia es una alternativa } \\
\text { educativa innovadora que ofrece diversas oportunidades } \\
\text { de estudio de manera no } \\
\text { presencial, con el apoyo de las TIC permitiendo facilitar } \\
\text { el acceso a la educación universitaria pública o privada, } \\
\text { con el } \\
\text { propósito de incrementar el nivel educativo de la } \\
\text { población y formar ciudadanos(as) comprometidos con } \\
\text { el desarrollo de cada país. }\end{array}$ \\
\hline $\begin{array}{l}\text { Virtual education: factors } \\
\text { that influence its expansion } \\
\text { in Latin America. }\end{array}$ & $\begin{array}{l}\text { Esta realidad exige a las instituciones no sólo una } \\
\text { actitud de cambio y apertura sino también, la } \\
\text { transformación de los modelos de enseñanza y de } \\
\text { gobernanza de las instituciones. }\end{array}$ \\
\hline $\begin{array}{l}\text { Educación en tiempos de } \\
\text { pandemia: reflexiones de } \\
\text { alumnos y profesores sobre } \\
\text { la enseñanza virtual } \\
\text { universitaria en España, } \\
\text { Italia y Ecuador }\end{array}$ & $\begin{array}{l}\text { El docente tiene que ser capaz de innovar, reflexionar y } \\
\text { transformar sus propuestas didácticas para responder a } \\
\text { las demandas sociales que vive el mundo en medio de } \\
\text { una crisis sanitaria, a la vez que se alcancen los } \\
\text { objetivos curriculares propuestos al inicio del curso. Por } \\
\text { otro lado, los docentes encuestados reconocen que es } \\
\text { necesario la promoción del pensamiento crítico y } \\
\text { reflexivo vinculado a la gestión estratégica de las TIC. }\end{array}$ \\
\hline $\begin{array}{l}\text { Entorno virtual para cocrear } \\
\text { recursos educativos digitales } \\
\text { en la educación superior }\end{array}$ & $\begin{array}{l}\text { Se expone que el } \\
\text { concepto de cocreación abre múltiples posibilidades de } \\
\text { gestar procesos enriquecidos, liderados y generados }\end{array}$ \\
\hline
\end{tabular}




\begin{tabular}{|c|c|}
\hline & $\begin{array}{l}\text { tanto por el } \\
\text { docente como por los estudiantes. Es una acción de } \\
\text { doble vía donde ambos perfiles proponen, producen y } \\
\text { distribuyen con } \\
\text { el fin de generar recursos educativos y compartir interés } \\
\text { y conocimiento en común. }\end{array}$ \\
\hline $\begin{array}{l}\text { El uso del Ambiente Virtual } \\
\text { de Aprendizaje entre el } \\
\text { profesorado de educación } \\
\text { superior: un análisis de } \\
\text { género. }\end{array}$ & $\begin{array}{l}\text { Aumento de uso de herramientas tecnológicas. No se } \\
\text { presentan diferencias significativas entre profesores y } \\
\text { profesoras el uso didáctico de la plataforma Moodle, así } \\
\text { como en la auto calificación del dominio de la misma. }\end{array}$ \\
\hline $\begin{array}{l}\text { Un reto en tiempos de } \\
\text { pandemia para la educación } \\
\text { médica en Cuba }\end{array}$ & $\begin{array}{l}\text { La educación médica en tiempos de COVID-19 } \\
\text { constituye un reto, por lo que ha tenido la necesidad de } \\
\text { reorganizar la educación de pregrado y posgrado para } \\
\text { garantizar la formación de nuestros profesionales, que } \\
\text { avalen futuros servicios médicos de calidad y excelencia }\end{array}$ \\
\hline $\begin{array}{l}\text { Tecnologias digitais de } \\
\text { comunicação e ensino } \\
\text { superior: contribuições da } \\
\text { teoria da atividade em } \\
\text { processos de ensino- } \\
\text { aprendizagem }\end{array}$ & $\begin{array}{l}\text { Los datos revelan que los procesos de enseñanza- } \\
\text { aprendizaje, en el } \\
\text { contexto de la Educación Superior, con el apoyo de las } \\
\text { tecnologías de la comunicación digital, basados en los } \\
\text { fundamentos } \\
\text { de la TA, presuponen una transformación cualitativa del } \\
\text { proceso y no solo una transposición de los métodos } \\
\text { de aprendizaje, inicialmente concebidos para una } \\
\text { enseñanza centrada en el docente, didácticamente } \\
\text { expositiva, y } \\
\text { que reduce la dimensión evaluativa del proceso de } \\
\text { enseñanza-aprendizaje a la mera realización de } \\
\text { ejercicios y aplicación } \\
\text { de conocimientos. }\end{array}$ \\
\hline $\begin{array}{l}\text { E-learning in } 15 \text { days. } \\
\text { Challenges and renovations } \\
\text { in Primary and Secondary } \\
\text { Education of the Republic } \\
\text { of Croatia during the } \\
\text { COVID-19 crisis. How have } \\
\text { we Introduced distance } \\
\text { Learning? }\end{array}$ & $\begin{array}{l}\text { Se establecen directrices, normas específicas y diversas } \\
\text { pautas que deben seguir los docentes, directores de } \\
\text { centro, familias y también los estudiantes para } \\
\text { alcanzar un modelo común de aprendizaje on-line } \\
\text { eficaz. }\end{array}$ \\
\hline $\begin{array}{l}\text { M- learning, un camino } \\
\text { hacia aprendizaje ubicuo en }\end{array}$ & $\begin{array}{l}\text { Se concluye que el M-learning, marca el camino hacia el } \\
\text { aprendizaje ubicuo, superando las limitantes } \\
\text { de tiempo y espacio. }\end{array}$ \\
\hline
\end{tabular}




\begin{tabular}{|c|c|}
\hline $\begin{array}{l}\text { la educación superior del } \\
\text { Ecuador }\end{array}$ & \\
\hline $\begin{array}{l}\text { El E-learning como } \\
\text { herramienta para la } \\
\text { educación continua: una } \\
\text { propuesta a la formación } \\
\text { profesional en el Ecuador }\end{array}$ & $\begin{array}{l}\text { los participantes dentro de aula, es la que sobresale } \\
\text { frente a la formación virtual, resaltando que el papel que } \\
\text { actualmente tiene la tecnología en la educación, es un } \\
\text { desafío para los adultos que aún tienen temor de salir del } \\
\text { método tradicional. }\end{array}$ \\
\hline $\begin{array}{l}\text { Plataformas digitales en la } \\
\text { educación a distancia en } \\
\text { México, una alternativa de } \\
\text { estudio en comunicación }\end{array}$ & $\begin{array}{l}\text { Se proporciona información sobre aspectos educativos } \\
\text { referentes a plataformas digitales y comunicativos desde } \\
\text { lo que son las revisiones sistemáticas siguiendo métodos } \\
\text { objetivos y sistematizados de evaluación, los datos e } \\
\text { información resultante serán de importancia ante la falta } \\
\text { de revisiones sistemáticas en el campo de la } \\
\text { comunicación educativa vinculada a las plataformas } \\
\text { digitales. }\end{array}$ \\
\hline $\begin{array}{l}\text { Hacia un nuevo concepto: } \\
\text { Plataformas Virtuales } \\
\text { Socioformativas (PVS) }\end{array}$ & $\begin{array}{l}\text { Se identifican nuevos espacios para trabajar la } \\
\text { educación en línea desde un enfoque socioformativo que } \\
\text { permita, tanto a } \\
\text { estudiantes como docentes, desarrollar el proceso de } \\
\text { enseñanza aprendizaje desde una perspectiva en la cual } \\
\text { se fortalece el empleo de proyectos transversales y } \\
\text { colaborativos. }\end{array}$ \\
\hline $\begin{array}{l}\text { Fortalecimiento de la } \\
\text { competencia TIC de } \\
\text { estudiantes de educación } \\
\text { superior en Ambientes } \\
\text { Virtuales de Aprendizaje }\end{array}$ & $\begin{array}{l}\text { Se concluye que en la investigación las competencias } \\
\text { TIC aportan flexibilidad e interactividad, permiten la } \\
\text { vinculación a una comunidad virtual de aprendizaje } \\
\text { donde se genera toda la comunicación entre los actores } \\
\text { del proceso }\end{array}$ \\
\hline
\end{tabular}

La indagación ha permitido identificar la importancia de la tecnología en el ámbito educativo y el hecho de establecer estrategias para la mejora de la calidad en el proceso de educación virtual, la implementación de estrategias didácticas para obtener aprendizajes significativos caracterizados por la flexibilidad e interactividad entre los participantes, identificar nuevos espacios para trabajar la educación en línea desde un enfoque socioformativo que permita desarrollar el proceso de enseñanza aprendizaje desde una perspectiva en la cual se fortalece el empleo de proyectos transversales y colaborativos, además de identificar las principales barreras para implementar 
la educación, como son las barreras culturales, asimismo plantear innovaciones tecnológicas para favorecer a las personas con discapacidad,

\section{Conclusiones}

Ha quedado comprobado que las tecnologías de la información y la comunicación contribuye de manera significativa diversas áreas y la educación no es la excepción, por ello es de vital importancia la identificación y adaptación de nuevas plataformas socioeducativas en el proceso enseñanza aprendizaje, de manera que se logre el objetivo educativo. Además se deben establecer estrategias tecnológicas y didácticas, tanto en los estudiantes como docentes para que el proceso sea flexible, dinámico y eficaz.

Al realizar una revisión de la metodológica ha permitido identificar diversos tipos de investigación de enfoque cuantitativo y cualitativo, de diversos niveles tanto descriptivos, exploratorios y correlaciónales. Al abarcar el tema de diferentes enfoques se considera un gran aporte a la investigación científica sobre la educación virtual, puesto que expone diversos ámbitos de la problemática. La población y muestra seleccionadas para los estudios presentados se encuentran inmersas en el ámbito educativo que ven con frecuencia la problemática. Además para el presente estudio se realizaron revisiones bibliográficas exhaustivas que permiten identificar las teorías y perspectivas que los autores.

En cuanto a los resultados se puede visualizar de manera más amplia las estrategias o medidas tomadas por los autores para la problemática planteada lo que permite obtener estrategias fundamentadas, que se pueden implementar en diversas instituciones del ámbito educativo, los artículos seleccionados promueven el análisis de la realidad y ofrece un acercamiento al tema de estudio, exponiendo nuevas propuestas sobre el fortalecimiento de la calidad educativa y como han resultado en otros contextos, exponiendo las 
limitaciones y deficiencias de cada estrategia para ser fortalecida y adecuada al contexto.

\section{Referencias}

Ahmadi, J. y Nourabadi, S. (2020). Implementation barriers in virtual education in Payame Noor University in Iran. Utopía Y Praxis Latinoamericana, 25(1), 202-210. https://produccioncientificaluz.org/index.php/utopia/article/view/32103 Aguirre Cardona, L. A., López Jaimes, J. E., \& Villamizar Gómez, D. F. (2019). Revisiones y reflexiones en la educación física: un camino de lo conceptual a lo investigativo en la escuela.

Avilés, J. (2015). Comunicar en la Sociedad Red. Barcelona: Editoral UOC. Basantes, A., Naranjo, M. y Ojeda, V. (2018). Metodología PACIE en la Educación Virtual: una experiencia en la Universidad Técnica del Norte. Formación Universitaria, 11(2), 35-44. http://dx.doi.org/10.4067/S071850062018000200035

Barros Bastidas, C., \& Turpo Gebera, O. (2018). Factors influencing the scientific production of university professors: a systematic review. Factores Que Influyen En La Producción Científica de Los Docentes Universitarios: Una Revisión Sistemática., 11(22), 225-234. http://10.0.85.43/pensam.v11i21-

1.276\%0Ahttps://ezproxy.uniandes.edu.co:8443/login?url=http://search.ebsc ohost.com/login.aspx ?direct=true $\& \mathrm{db}=$ fua $\& A N=141223533 \&$ lang=es $\&$ site $=$ ehost-live

Cano, J. Domínguez, A. y Ricardo, C. (2018). Fortalecimiento de la competencia TIC de estudiantes de educación superior en Ambientes Virtuales de Aprendizaje. Revista espacios. 39. https://www.revistaespacios.com/a18v39n25/a18v39n25p35.pdf 
Castro, M. (2020). Tecnologias digitais de comunicação e ensino superior: contribuições da teoria da atividade em processos de ensino - aprendizagem. 49 (49). Revista lusófona de educação. https://revistas.ulusofona.pt/index.php/rleducacao/article /view/7453 Chinchay, S., Moreno-Quispe, L., Santa Cruz, A., Zerga, J. y Cango J. (2021) Perspectivas de la Educación Superior en los Entornos Virtuales en Perú. Revista Ibérica de Sistemas e Tecnologias de Informação. 39. Pp 219 228.http://www.risti.xyz/issues/ristie39.pdf.

Deliyore, M. (2021). Redes como espacio de comunicación para la educación virtual de estudiantes con discapacidad en Costa Rica en tiempos de pandemia. Historia Y Comunicación Social, 26(Especial), 75-85. https://doi.org/10.5209/hics.74243

Del Prete, A., y Cabero, J. (2020). El uso del Ambiente Virtual de Aprendizaje entre el profesorado de educación superior: un análisis de género. Revista de Educación a Distancia (RED), 20(62). https://doi.org/10.6018/red.400061

Díaz, P., Andrade, Y., Hincapié, A. y Uribe, A. (2021). Análisis del proceso metodológico en programas de educación superior en modalidad virtual. Revista de Educación a Distancia (RED), 21(65). https://doi.org/10.6018/red.450711

Freire, R., Vera, N. y Díaz, J. (2019) Marco legal de la educación virtual a distancia hacia su implementación en el contexto latinoamericano. Revista Ibérica de Sistemas e Tecnologias de Informação. 25. Pp 87-102.

García-Ruiz, R. y Pérez-Escoda, A. (2020). Comunicación y Educación en un mundo digital y conectado. Presentación, Icono 14, 18 (2), 1-15. Doi: 10.7195/ri14.v18i2.1580

Gómez-Hurtado, I., García-Rodríguez, M., González, I., y Coronel, J. (2020). Adaptación de las Metodologías Activas en la Educación Universitaria en 
Tiempos de Pandemia. Revista Internacional De Educación Para La Justicia

Social, 9(3), 415-433. https://doi.org/10.15366/riejs2020.9.3.022

González-García, S., Casadelvalle, I., Octavio, M., Fortún, T., Mezquía, N., y Melón, R. (2020). Un reto en tiempos de pandemia para la educación médica en Cuba. Educación Médica Superior, 34(3), e2457. http://scielo.sld.cu/scielo.php?script=sci_arttex t\&pid=S0864$21412020000300016 \& \operatorname{lng}=$ es\&tlng=es.

Grosso, J. (2017). NTICS, educación virtual, lógicas de la comunicación social y "sociedad del conocimiento": anotaciones sobre el contexto y la responsabilidad social de la educación superior a distancia. Revista Diálogo Educacional, 4(13), 69-78.

Hernández, A., Carro, E. y Martínez, I. (2019). Plataformas digitales en la educación a distancia en México, una alternativa de estudio en comunicación. Revista de Educación a Distancia (RED), 19(60). https://doi.org/10.6018/red/60/07

Herrera-Tapia, J. y Navia, M. (2020), Las tecnologías de la información: Aliado y soporte para las organizaciones en un mundo en crisis, RISTI: Revista Ibérica de Sistemas e Tecnologías de Informação, https://cat.biblioteca.ua.es

/permalink/34CVA_UA/1htbb30/cdi_proquest_journals_239453670

Hodges, C., Moore, S., Lockee, B., Trust, T. y Bond, A. (2020). The difference between emergency remote teaching and online learning. Educause Review. https://er.educause.edu/articles/2020/3/the-differencebetween-emergency-remote-teaching-and-online-learning Lagos, G., Mora, K., Mejía, D., Peláez, R. y Peláez J. (2018) M- learning, un camino hacia aprendizaje ubicuo en la educación superior del Ecuador. Revista Ibérica de Sistemas e Tecnologias de Informação. 18. 47-59. 
Manrique-Losada, B. Zapata, M. y Arango, S. (2020). Entorno virtual para cocrear recursos educativos digitales en la educación superior. Campus Virtuales, 9(1), 101-112.

Navarrete, G., Morán, C., Guamán, M., Arteaga, M., Torres, J.() El E-learning como herramienta para la educación continua: una propuesta a la formación $\begin{array}{llllll}\text { profesional en } & \text { el } & \text { Ecuador. } & \text { PP. }\end{array}$ https://www.proquest.com/openview/f4b193b46ccb16a2d

f948137e69c2b20/1?pq-origsite $=$ gscholar \&cbl=1006393

Prata, D., Barbato, S. y Gonzalez M. (2020) Ambientes virtuales de aprendizaje y producción de identidad en la formación inicial docente. Digital Education Review . 38. https://revistes.ub.edu/index.php/der/article/view/27939/pdf

Rodríguez, C., y Juanes, B. (2021). Implementación de una estrategia didáctica para la interactividad en ambientes virtuales para el posgrado en la Educación Superior. Revista Universidad y Sociedad, 13(1), 307-316. https://rus.ucf.edu.cu/index.php /rus/article/view/1927

Sianes, A., y Sánchez, E. (2020). E-learning in 15 days. Challenges and renovations in Primary and Secondary Education of the Republic of Croatia during the COVID-19 crisis. How have we Introduced distance Learning?. Revista Española de Educación Comparada, 0(36), 181-195. doi:https://doi.org/10.5944/reec.36.2020.27637

Tejedor, S., Cervi, L., Tusa, F. y Parola, A. (2020). Educación en tiempos de pandemia: reflexiones de alumnos y profesores sobre la enseñanza virtual universitaria en España, Italia y Ecuador. Revista Latina de Comunicación Social, 78, 1-21. https://www.doi.org/10.4185/RLCS-2020-1466

Tobon, B., Tobón, S., Veytia-Bucheli, M. y Escudero, A. (2018) Hacia un nuevo concepto: Plataformas Virtuales Socioformativas (PVS). Revista 
espacios. 39 (53) http://www.revistaespacios.com/cited2017/cited201727.html

Varas-Meza, H. Suárez-Amaya, W. López-Valenzuela, C. y ValdésMontecino M. (2020). Virtual education: factors that influence its expansion in Latin America. http:doi.org/10.5281/zenodo.4292698

Vergara, E., Vergara, R., Alvarez, M., Camacho, L., y Galvez, J. (2020). Educación médica a distancia en tiempos de COVID-19. Educación Médica Superior, $34(2)$, e2383.

http://scielo.sld.cu/scielo.php?script=sci_arttext\&pid=S0864-

$21412020000200025 \& \operatorname{lng}=$ es\&tlng=es.

Villegas, L., Walle-Vazquez, M. y Álvarez-Aros, E. (2020), Uso de las tecnologías de la información en los procesos de toma de decisiones: un análisis bibliométrico, Revista Venezolana de Gerencia. https://www.researchgate.net/

publication/348885065_Uso_de_las_tecnologias_de_la_informacion_en_los _procesos_decisorios_un_analisis_bibliometrico 